\title{
DIGITAL WATERMARKING WITH A NEW ALGORITHM
}

\author{
Afroja Akter ${ }^{1}$, Muhammad Ahsan Ullah ${ }^{2}$ \\ ${ }^{1,2}$ Department of Electrical and Electronic Engineering, Chittagong University of Engineering and Technology, \\ Chittagong, Bangladesh
}

\begin{abstract}
Everyday millions of data need to transmit through a distinct channel for various purposes; as a result there is a certain chance of third person interruption on that data. In this regards digital watermarking is one of the best solution. This paper proposes a new embedding algorithm (NEA) of digital watermarking. The algorithm is performed for digital image as data. The performance is compared for NEA and well established Cox's modified embedding algorithm. The watermarking is based on discrete wavelet transforms (DWT) and discrete cosine transforms (DCT). The acceptance of the new algorithm is measured by the two requirements of digital watermarking. One is imperceptibility of the watermarked image, measured by peak signal to noise ratio (PSNR) in dB; another one is robustness of the mark image, measured by correlation of original mark image and recovering mark image. Here a $512 \times 512$ gray scale "Lena" and "Cameraman's" image is taken as host images, and a 128×128 gray scale image is taken as mark image for 2 level of DWT. The simulation results for different attacking conditions such as salt and pepper attack, additive white Gaussian noise (AWGN) attack, jpg compression attack, gamma attack, histogram attack, cropping attack, sharpening attack etc. After different attacks the changing tendency PSNR for both algorithms are similar. But the mean square error (MSE) value of NEA is always less than Cox's modified algorithm, which means that after embedding the changes of the host image property lower for NEA than Cox's algorithm. From the simulation results it can be said that NEA will be a substitute of modified Cox's algorithm with better performance.
\end{abstract}

Keywords: Digital watermark, DWT, DCT, Cox's modified algorithm, Lena image, Cameraman image, AWGN, JPG, salt and pepper attack, PSNR, correlation, MSE.

\section{INTRODUCTION}

Digital watermarking is a scheme where a secret data, it may an image, audio or video, embedded in a host data such a way that it remains on it as long as the perceptible quality of the content in an acceptable level [1]. From the last decade digital watermarking is best choice for data security. Digital watermarking may classified as (1) blind or (2) informed. This paper only focused on informed digital watermarking, where the mark signal extracted using the host data. Digital watermarking procedure mainly classified into three steps [14]
$>$ Embedding
$>$ Distribution
$>$ Extraction or Detection

Embedding is the process where a secret data i.e. either an image or an audio or video is embedded into a host data which may an image or an audio or video, using a key. Then this embedded data is broadcast or distributed through a lossless or lousy channel, which is known as distribution. The third step is depending on author, when the existence of the mark signal can be detected using the host data with known key is known as detection. But when the mark data is recover from the embedded data using the known host data and key is known as extraction. The process is shown in figure 1 .

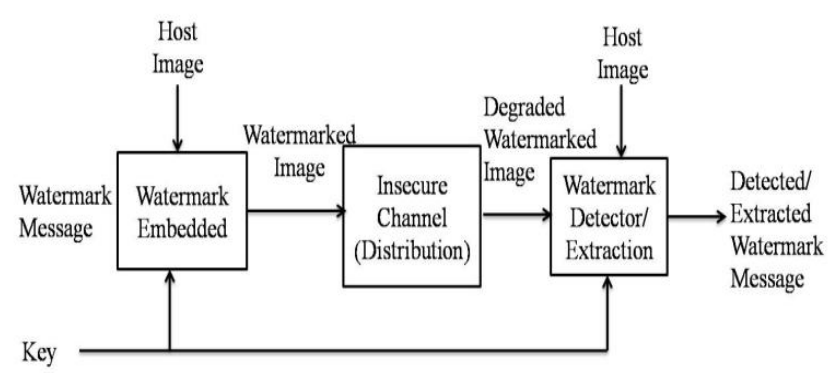

Fig 1: Digital watermarking system.

Furthermore digital watermarking is based on two techniques

$>$ Spatial domain based [2]

$>$ Transform domain based

The most common spatial domain based technique is least significant bit (LSB) [2-4, 13] method. The proposed technique is based on transform domain. Again the transform domain based digital watermarking can be classified into discrete wavelet transforms (DWT) [5-10, 12, 14], discrete Cosine transforms (DCT) $[5,7,11,15]$. Multi resolution properties is the most important characteristics of DWT based digital watermarking, as for the specified region can be selected for data embedding. Every image can be divided into 
two specific regions: (1) low frequency region and (2) high frequency region, and these regions can be sub-divided in case of DWT based digital watermarking. DCT divide the image in three regions: (1) low frequency region, (2) mid-frequency region and (3) high frequency region. It is easy to select a distinct region to embed a data in case of DCT based digital watermarking. This paper based on both of these technique, thus it can provide better performance.

A $512 \times 512$ gray scale image is taken as host image, and a $128 \times 128$ gray scale image is taken as mark image. After 2level of DWT of host image the mark is embedded on a selected sub-band. This paper considers the sub-bands from a high frequency region of DWT, and then selects the comparative low frequency or high co-efficient sets for DCT. At lower level of DWT based digital watermarking it is challenging to embed the mark image into the host image without hampering its perception level, thus here this situation is tested.

Acceptance of the proposed algorithm is measured by two most important requirements of the digital watermarking. One of them is imperceptibility of the embedded data, measured by peak signal to noise ratio (PSNR) in $\mathrm{dB}$, and remaining one is robustness of the recovering data, measured by the correlation of original mark data and recover data. Greater PSNR is desired, as it means the perception level of the host image is little bit interrupted by the mark image. And a better correlation (maximum $100 \%$, i.e. 1) measured that the mark image is not interrupted by the attacks in a broadcasting channel. Another parameter known as mean square error (MSE) also considering. As less MSE indicates that the mark image interrupts the host image less. i.e. less MSE is acceptable. This paper evaluate the technique considering different attacks like salt and pepper attack, additive white Gaussian noise (AWGN) attack, jpg compression attack, gamma attack, histogram attack, cropping, sharpening attack, rotational attack etc. From simulation results it can be decided that the new algorithm is accepted with comparing Cox's modified algorithm even at different attack condition.

This paper is arranged as following sections; in section two different parameters for digital watermarking are discussed. The hybrid technique of digital watermarking is discussed in section three. Simulation results are presented in section four.

\section{PERFORMANCE MEASURE}

Digital watermarking has certain requirements. To measure these requirements different image quality metrics are used. This paper is using peak signal to noise ratio (PSNR) to measure the amount of visual quality degradation between original and watermarked image. This criterion is very well known as imperceptibility of the embedded image [7]. This paper also measures the robustness of the mark image.
Robustness is measured by correlation of original mark image and recovered mark image.

\subsection{Peak Signal-To-Noise Ratio (PSNR)}

To measure the quality of reconstruction of lossy compression PSNR is frequently used [7, 13]. And it is the ratio of the maximum possible power of a host image to the power of corrupting noise that affects the fidelity of its representation. It can define by the following equation:

$$
P S N R_{d B}=20 \times \log _{10}\left(\frac{M A X}{\sqrt{M S E}}\right)
$$

Where MAX represents the maximum possible pixel value in the host image and MSE is shows the mean square error in embedded image.

MSE is measured from the host image and noisy approximation of it [13]. MSE can be defined by the equation (2.2)

$$
M S E=\left(\frac{1}{m \times n}\right) \sum_{k=1}^{m} \sum_{l=1}^{n}\left(f(k, l)-f^{\prime}(k, l)\right)^{2}
$$

Where, $f(k, l)$ is host image and $f^{\prime}(k, l)$ is watermarked/embedded image.

\subsection{Robustness}

Robustness measure the tolerance of the technique after experiencing different attacks, it is measured for the mark image. I.e. distributing of the embedded image through a loosy channel how much it experiences the degradation of mark image is measured by robustness. The measuring tool of it is correlation of the original mark image and recover mark image. Correlation of two images can be found from following equation.

$$
\text { Corr. }=\frac{\sum_{i} \sum_{j}\left\{w(i, j)-w_{\text {avg. }}(i, j)\right\}^{*}\left\{w^{\prime}(i, j)-w_{\text {avg. }}^{\prime}(i, j)\right\}}{\sqrt{\left.\left(\sum_{i} \sum_{j}\left\{w(i, j)-w_{\text {avg. }}(i, j)\right\}^{2}\right)\left(\sum_{i} \sum_{j}\left\{w^{\prime}(i, j)-w_{\text {avg. }}^{\prime}(i, j)\right\}^{2}\right)\right)}}
$$

where $w(i, j)$ and $w^{\prime}(i, j)$ ' are the original and extracted/recover watermark image respectively.

\section{HYBRID TECHNIQUE}

This paper discusses the digital watermarking technique based on transform domain. Most popular transform domain techniques included: discrete wavelet transforms (DWT) and discrete cosine transforms (DCT), separately these techniques having some merits and demerits, but combining both of these 
can provides better performance. In this regard this paper evaluates the new algorithm based on hybrid technique. In this paper this hybrid digital watermarking technique is discussed in two steps:

$>$ Digital watermarking embedding technique.

$>$ Digital watermarking extracting technique.

Both steps are discussed as follows:

\subsection{Digital Watermarking Embedding Technique:}

Using DWT a $512 \times 512$ host image (here gray scale "Lena" or "Cameraman") is sub-divided into 4-subbands. Figure 3 represents the 1st level of DWT decomposition of host image. Where LL, HL, LH, HH are 4 sub-bands. Here LL represents the high scale low frequency co-efficient set, HL represents the horizontal details of low scale high frequency set, LH represents the vertical details of low scale high frequency set, $\mathrm{HH}$ represents the diagonal details low scale high frequency set of the host image.

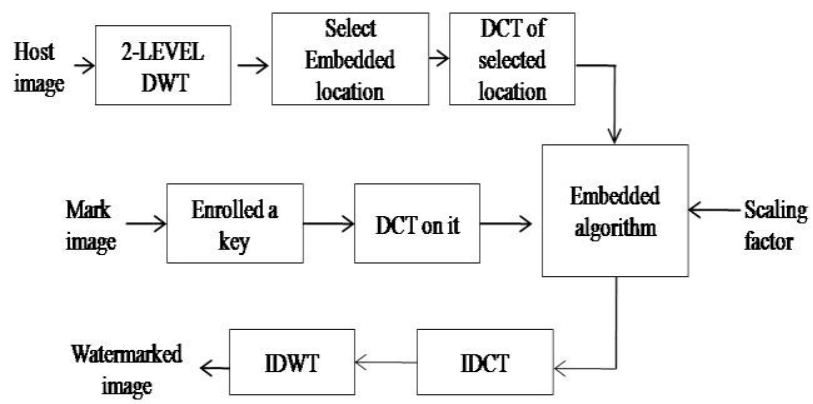

Fig 2: Flow chart of the embedding procedure.

Then using 2 dimensional of DWT on HL of the host image, 2nd level decompose image can be found as shown in figure 4.

After the 2nd level decomposition of the host image then the comparative low frequency components denote as $\mathrm{HL}_{\mathrm{LL} 2}$ is select for 2 dimensional DCT. It is common practice to select the mid frequency region for embedding purposes during DCT based digital watermarking for robustness, but having chance to degrade the perceptibility of the host image. To trade of the imperceptibility and robustness this paper focused on the low frequency region after 2 dimensional DCT of the host image. Then a $128 \times 128$ gray scale image as a mark is embedded on the selected region after enrolled a key and DCT on it. With scaling factor 0.9 the mark image is embedded into the host image using the new hybrid algorithm and Cox's modified algorithm as in equation 3.1 and equation 3.2 respectively

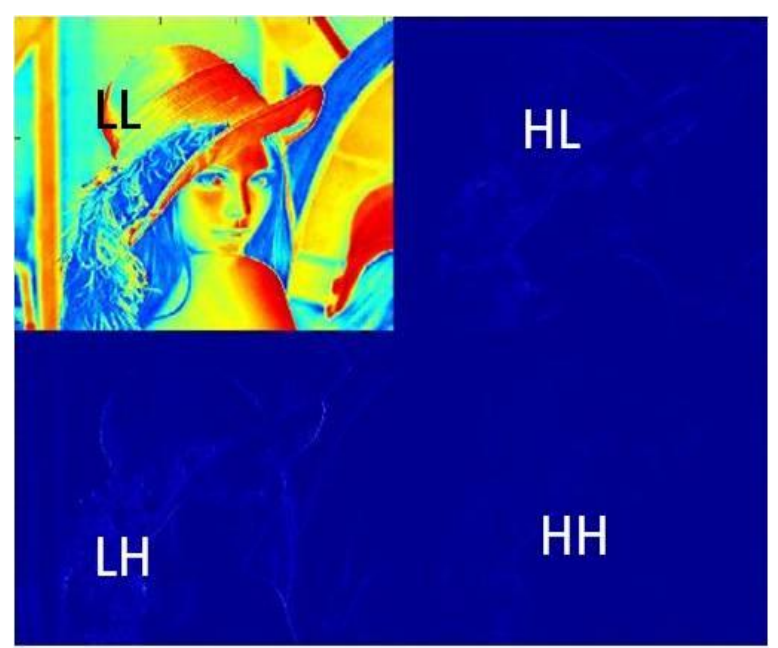

Fig 3: 1st level DWT decomposition of "Lena" image.

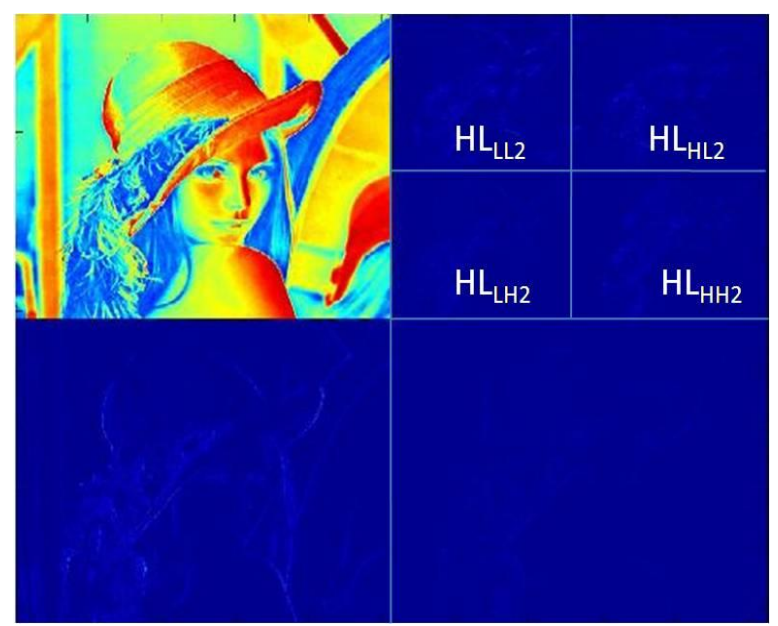

Fig 4: 2nd level DWT decomposition of "Lena" image.

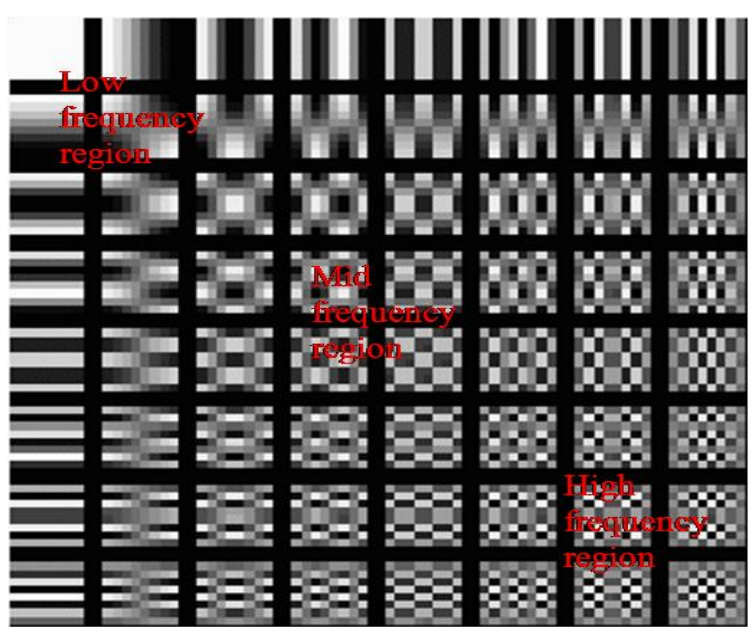

Fig 5: DCT region of an image 


$$
\begin{aligned}
& f^{\prime}(m, n)=f(m, n)+\alpha \times w \frac{f(m, n)-f_{\text {avg }}(m)}{\sqrt{\left|f(m, n)^{2}-f_{\text {avg }}(m)^{2}\right|}} \\
& f^{\prime}(\boldsymbol{m}, \boldsymbol{n})=f(\boldsymbol{m}, \boldsymbol{n})+\alpha \times w
\end{aligned}
$$

Where $f(m, n)$ is the host image, $f_{\text {avg. }}(m)$ is mean value of the host image, $w$ is original mark image.

Here from Cox's modified algorithm it has been shown that the mark image totally independent on host image, thus the imperceptibility is degraded sharply when embedded or watermarked image is experienced different attacks from without attack condition. But from the new algorithm it is noticed that the mark image is multiplied with the absolute deviation of host image. Thus here degradation rate is slow.

\subsection{Digital Watermarking Extraction Technique:}

The extraction technique is depicts in figure 6. To extract the mark image from the embedded or watermarked image the original $512 \times 512$ image is used, hence this is an informed digital watermarking technique.

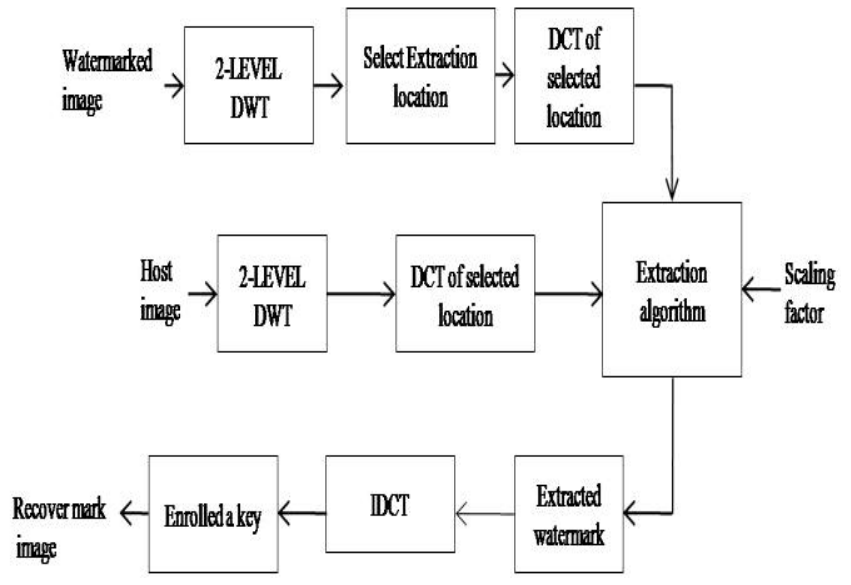

Fig 6: Flowchart of extracting procedure

First of all the watermarked image is decomposed into 2-level of DWT, then the extraction location is selected. Then DCT is done on the selected sub-bands. Similarly the host image is first decomposing into 2-level DWT and then DCT on the selected sub-band. Then the mark image is extracted using the new algorithm and Cox's modified algorithm respectively as bellows in equation 3.3 and equation 3. 4 :

$$
w^{\prime}=\frac{\left[f^{\prime}(m, n)-f(m, n)\right] \sqrt{\left|f(m, n)^{2}-f_{\text {avg }}(m)^{2}\right|}}{\alpha\left[f(m, n)-f_{\text {avg }}(m)\right]}
$$

$$
w^{\prime}=\frac{f^{\prime}(m, n)-f(m, n)}{\alpha}
$$

Where $w^{\prime}$ is recover image. Then IDCT is done on the extracted watermark and the recover mark image is found enrolled the secret key.

\section{RESULTS}

To evaluate the performance of the new algorithm and comparative result, here $512 \times 512$ Lena image and a $512 \times 512$ Cameraman image are used while a $128 \times 128$ gray scale image is used as mark image. For simulation considering scaling factor is 0.9 .

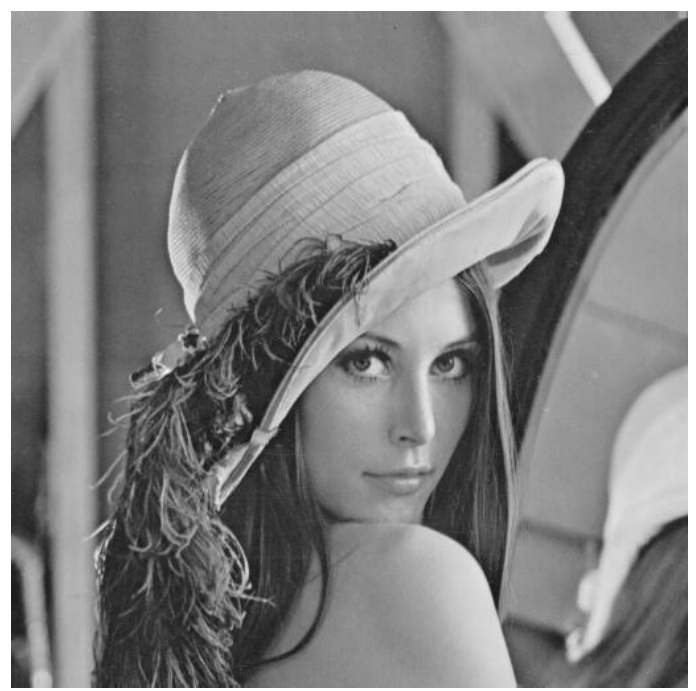

Fig 7: Host Lena image

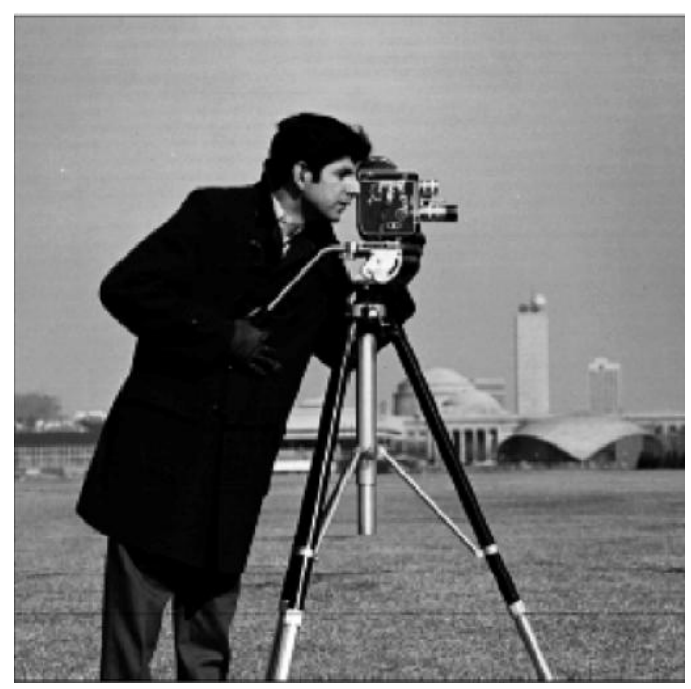

Fig 8: Host Cameraman image. 
Table 1: PSNR, MSE and Correlation Values for Lena image

\begin{tabular}{|l|c|c|c|c|c|c|}
\hline & \multicolumn{3}{|c|}{ New algorithm } & \multicolumn{3}{c|}{ Cox's modified algorithm } \\
\hline \multicolumn{1}{|c|}{ Attack } & PSNR(dB) & MSE & Correlation & PSNR(dB) & MSE & Correlation \\
\hline No attack & 27.87 & 98.08 & 1.00 & 27.69 & 102.14 & 1.00 \\
\hline Salt and pepper & 28.59 & 82.97 & 0.85 & 27.73 & 101.08 & 0.91 \\
\hline AWGN & 28.12 & 92.47 & 0.77 & 27.65 & 103.11 & 0.85 \\
\hline Jpg compression & 28.62 & 82.33 & 0.99 & 27.75 & 100.63 & 0.99 \\
\hline Gamma Correction & 23.73 & 253.9 & 0.85 & 23.72 & 255.00 & 0.88 \\
\hline Histogram & 27.62 & 103.64 & 0.86 & 27.60 & 104.10 & 0.88 \\
\hline Cropping & 28.68 & 81.22 & 0.96 & 27.80 & 99.56 & 0.97 \\
\hline Sharpening & 27.80 & 99.53 & 0.83 & 27.76 & 100.40 & 0.84 \\
\hline Speckle Noise & 27.13 & 116.03 & 0.28 & 26.94 & 121.19 & 0.37 \\
\hline Median filter & 29.41 & 68.81 & 0.81 & 28.41 & 86.41 & 0.85 \\
\hline Scaling & 31.26 & 44.81 & 0.29 & 30.92 & 48.48 & 0.34 \\
\hline
\end{tabular}

Table 2: PSNR, MSE and Correlation Values for Cameraman image

\begin{tabular}{|l|c|c|c|c|c|c|}
\hline & \multicolumn{3}{|c|}{ New algorithm } & \multicolumn{3}{c|}{ Cox's modified algorithm } \\
\hline Attack & PSNR(dB) & MSE & Correlation & PSNR(dB) & MSE & Correlation \\
\hline No attack & 28.13 & 99.97 & 1.00 & 28.03 & 102.15 & 1.00 \\
\hline Salt and pepper & 29.01 & 81.5195 & 0.72 & 28.95 & 82.67 & 0.87 \\
\hline AWGN & 28.77 & 86.21 & 0.58 & 28.72 & 87.19 & 0.80 \\
\hline Jpg compression & 29.04 & 81.09 & 0.95 & 28.97 & 82.33 & 0.98 \\
\hline Gamma & 25.11 & 200.06 & 0.55 & 25.07 & 202.16 & 0.66 \\
\hline Histogram & 29.49 & 73.09 & 0.57 & 29.45 & 73.67 & 0.81 \\
\hline Cropping & 29.08 & 80.21 & 0.89 & 29.02 & 99.56 & 0.97 \\
\hline Sharpening & 32.92 & 34.92 & 0.69 & 35.89 & 16.73 & 0.89 \\
\hline Speckle Noise & 28.25 & 97.28 & 0.18 & 28.22 & 97.89 & 0.31 \\
\hline Median filter & 29.14 & 79.10 & 0.53 & 29.22 & 77.65 & 0.79 \\
\hline Scaling & 29.17 & 78.65 & 0.10 & 29.94 & 65.80 & 0.14 \\
\hline
\end{tabular}

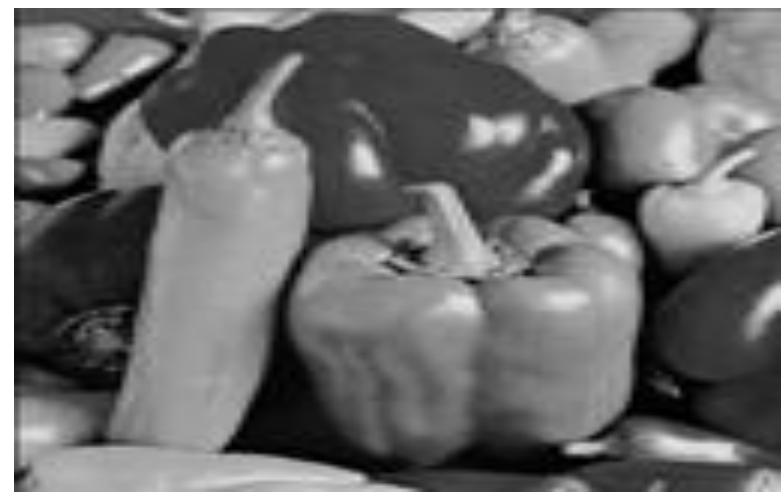

Fig 9: Watermark image.
Figure 7 is Lena image and figure 8 is Cameraman image. In this paper both of these image are consider as host image. And figure 9 represents the watermark image. Table 1 shows different results for both new algorithm and Cox's modified algorithm for Lena image, whereas table 2 represents results for Cameraman image. From both table it can be said that the PSNR value of the watermarked image is slightly degraded from the original image at different attacks whereas this is sharply degraded for Cox's modified algorithm. Here salt and pepper attack, additive white Gaussian noise (AWGN) attack, jpg compression attack, gamma attack, histogram attack, cropping attack, sharpening attacks are consider for simulation. For salt and pepper attack the PSNR value for the Cox's modified algorithm is increased. This phenomenon is 
also noticed for the NEA. But MSE value of the Cox's modified algorithm is greater than the NEA, which means that the host image is less hampered for NEA than the Cox's algorithm. Similar criterion is noticed for other attacks like AWGN, Jpg compression, cropping, median filter and scaling attacks. But in all cases the PSNR of the new embedding algorithm (NEA) is higher than the Cox's modified algorithm. In case of correlation the NEA and Cox's modified algorithm having almost similar value. Thus considering all conditions this paper appreciates the new algorithm for digital watermarking.

\section{CONCLUSIONS}

An informed digital watermarking based on DWT-DCT technique with a new algorithm is presents in this paper. This paper also shows the comparative results of Cox's modified algorithm and new algorithm for Lena and Cameraman image. The PSNR changing criterion is similar for both new embedding algorithm (NEA) and Cox's modified algorithm. Although in all cases PSNR of the NEA is higher than the Cox's algorithm. The MSE value indicates that the NEA can embed the mark image into the host image with less degradation than the Cox's algorithm. From all simulation results this paper appreciates the performance of new embedding algorithm (NEA) and it can be said that the NEA will be a substitute of modified Cox's algorithm with better performance.

\section{REFERENCES}

[1]. Jonathan K. Su, Frank Hartung, Bernd Girod,” Digital Watermarking of Text, Image, and Video Documents", Telecommunications Laboratory University of ErlangenNuremberg Erlangen, Germany.

[2]. Ibrahim Nasir, Ying Weng, Jianmin Jiang," A New Robust Watermarking Scheme for Color Image in Spatial Domain", School of Informatics, University of Bradford, UK

[3]. R.G.van Schyndel, A.Z.Tirkel, C.F.Osborne, " $A$ DIGITAL WATERMARK ",Department of Physics, Monash University, Clayton, 3168, Australia. Scientific Technology, 21 Walstab St, E. Brighton, 3187, Australia

[4]. Mr. Rohith.S1, Dr. K.N.hari bhat2," A Simple Robust Digital Image Watermarking against Salt and Pepper Noise using Repetition Codes ",Nagarjuna College of Engineering and Technology, Bengaluru, Karnataka, India.

[5]. Shital Gupta, Dr Sanjeev Jain, "A Robust Algorithm of Digital Image Watermarking Based on Discrete Wavelet Transform", Department of Computer Science and Engineering, LNCT, Bhopal.

[6]. Nidhi Bisla, Prachi Chaudhary, "Comparative Study of DWT and DWT-SVD Image Watermarking Techniques", International Journal of Advanced Research in Computer Science and Software Engineering", Volume 3, Issue 6, June 2013

[7]. M.A. Mohamed and A.M. El-Mohandes, "Hybrid DCTDWT Watermarking and IDEA Encryption of Internet
Contents", International Journal of Computer Science Issues, Vol.9, Issue 1, No 2, January2012

[8]. Emir Ganic, Ahmet M. Eskicioglu,"Robust DWT-SVD Domain Image Watermarking: Embedding Data in All Frequencies".

[9]. Gaurav Bhatnagar, Balasubramanian Raman," A new robust reference watermarking scheme based on DWT-SVD", Department of Mathematics, Indian Institute of Technology Roorkee, Roorkee - 247667, India

[10]. Praful Saxena, Shanon Garg and Arpita Srivastava," DWT-SVD Semi-Blind Image Watermarking Using High Frequency Band", 2nd International Conference on Computer Science and Information Technology (ICCSIT'2012) Singapore April 28-29, 2012.

[11]. Mr.D.V.N.Koteswara Rao,Y.Madhuri, S.V.Rajendra Kumar, Y.V.Suresh Babu, " Robust Image Watermarking using DCT \& Wavelet Packet Denoising ", International Journal of Scientific \& Engineering Research Volume 3, Issue5, May2012.

[12]. Corina Nafornita and Alexandru Isar, "Application of discrete wavelet transform in watermarking", Politehnica" University of Timisoara, Romania.

[13]. Koushik Pal, G. Ghosh1 and M. Bhattacharya, " A Novel Digital Image Watermarking Scheme for Data Security Using Bit Replacement and Majority Algorithm Technique", Institute of Radio Physics and Electronics, University of Calcutta, Kolkata Indian .

[14]. Ms. T Mita Kumari, "Image Adaptive Watermarking Using Wavelet Transform", Department of Electrical Engineering, National Institute of Technology, Rourkela 769008, 2007.

[15]. Abrar Ahmed Syed, "Digital Watermarking", The University of Texas, Arlington.

\section{BIOGRAPHIES}

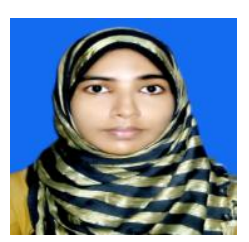

Afroja Akter, Graduated in Electrical and Electronic Engineering, From Chittagong University of Engineering and Technology, Chittagong, Bangladesh in September 2013. Her research interests are in image processing, VLSI, and Renewable energy.

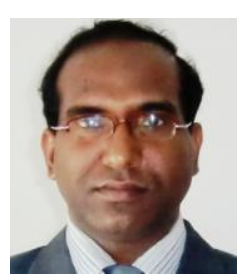

Muhammad Ahsan Ullah received B.Sc. in Engineering degree in Electrical and Electronic Engineering from Chittagong University of Engineering and Technology, Bangladesh in 2002, M.E. degree in Electronic and Information Engineering from Kyung Hee University, Republic of Korea in 2007 and $\mathrm{PhD}$ degree in Electrical Engineering at Nagaoka University of Technology at 2011. He is interested in Signal processing, Coding Theory, threshold decoding, MCCDMA, OFDMA, MIMO, and Image Processing. 